\title{
Cough and hypereosinophilia due to FIP1L1-PDGFRA fusion gene with tyrosine
} kinase activity

\author{
K.F. Chung*, M. Hew*, J. Score" , A.V. Jones", A. Reiter", N.C.P. Cross" and B.J. Bain ${ }^{+}$
}

ABSTRACT: Eosinophil-associated conditions, such as asthma and eosinophilic bronchitis, have been associated with chronic persistent cough, usually responding to corticosteroid therapy.

This case study reports a case of persistent cough associated with gastro-oesophageal reflux (GOR) and hypereosinophilia. Treatment of GOR with proton pump inhibitors and fundoplication did not control the cough. However, high dose prednisolone, but not inhaled corticosteroids, did.

The presence of the FIP1L1-PDGFRA fusion gene in myeloid cells was confirmed by fluorescence in situ hybridisation analysis using $\mathrm{CHIC2}$ deletion as a surrogate marker. The cough and other disease features were subsequently suppressed by the tyrosine kinase inhibitor, imatinib.

This is the first case of persistent cough caused by hypereosinophilic syndrome characterised by FIP1L1-PDGFRA fusion gene and aberrant tyrosine kinase activity.

KEYWORDS: Cough, fusion gene, hypereosinophilic syndrome, imatinib, tyrosine kinase

0 hronic cough is a common clinical problem [1, 2]. Asthma, post-nasal drip or rhino-sinusitis, and gastro-oesophageal reflux are the most common causes of chronic cough [3], but in many patients, no cause can be identified despite thorough investigations and empirical treatment [4, 5]. Conditions associated with eosinophils in the airways, such as asthma and eosinophilic bronchitis, have been associated with chronic cough and treatment with corticosteroids, which suppress eosinophils, is usually effective in controlling such cough. This study describes a patient with persistent cough associated with FIP1L1-PDGFRA positive hypereosinophilia, a condition that has been described recently [6]. This cough was controlled by highdose oral corticosteroid therapy and also by a specific inhibitor of this acquired, constitutively active tyrosine kinase, imatinib.

\section{CASE REPORT}

A previously well 54-yr-old male scientist, an expert in bomb disposal, developed a chronic cough in 2001. He was investigated using spirometry, computed tomograms of the lungs and an endobronchial biopsy; all were normal.

For editorial comments see page 1.
However, his eosinophil count was $1.7 \times 10^{9} \cdot \mathrm{L}^{-1}$. $\mathrm{He}$ was treated with inhaled corticosteroids for 3 months and a week's course of prednisolone without benefit. He complained of regular episodes of gastro-oesophageal reflux and a 24-h recording of oesophageal $\mathrm{pH}$ showed significant reflux of gastric acid. Treatment with proton pump inhibitors for up to 6 weeks and a Nissen fundoplication did not resolve his cough, although his symptoms of reflux disappeared after surgery.

In 2003, he was referred to the Royal Brompton Hospital because of intractable cough, which prevented him from working. He was producing a small amount of clear sputum. He also complained of diarrhoea and night sweats, and had lost $15 \mathrm{~kg}$ in weight in the preceding $2 \mathrm{yrs}$. He had stopped smoking 15 yrs previously with a 15 pack-yr history. Clinical examination was normal. Blood eosinophil count was $5.0 \times 10^{9} \cdot \mathrm{L}^{-1}$ and immunoglobulin $\mathrm{E}$ was $61 \mathrm{IU} \cdot \mathrm{mL}^{-1}$ (normal range 3-150 IU $\cdot \mathrm{mL}^{-1}$ ); no anti-neutrophil cytoplasmic antibodies were detected. Computed tomography of the lungs showed thickening of intrapulmonary airways with distal airway plugging. Forced expiratory volume in one second, lung volumes and carbon monoxide gas transfer

\section{AFFILIATIONS}

*National Heart and Lung Institute, Imperial College and Royal Brompton Hospital,

+St Mary's Hospital Campus of Imperial College, London, and, "Wessex Regional Genetics Laboratory, Salisbury District Hospital, Salisbury, UK.

•III Medizinische Universitätsklinik, Fakultät für Klinische Medizin Mannheim der Universität Heidelberg, Heidelberg, Germany.

CORRESPONDENCE

K.F. Chung

National Heart and Lung Institute

Dovehouse Street

London SW3 6LY

UK

Fax: 442073518126

E-mail: f.chung@imperial.ac.uk

Received:

August 022005

Accepted after revision:

October 132005

European Respiratory Journal

Print ISSN 0903-1936

Online ISSN 1399-3003 
were normal, but he had a sensitive cough response to inhaled capsaicin. Endobronchial biopsy showed goblet cell hyperplasia and eosinophilic stromal inflammation. Bronchoalveolar lavage fluid contained $25 \%$ eosinophils. Because of his extensive travels in tropical countries, stool examination and serological investigations were carried out and excluded parasitic infestations. A course of ivermectin had no effect on his cough or eosinophilia. Abdominal computed tomography revealed hepatosplenomegaly with a $16-\mathrm{cm}$ long spleen and a craniocaudal liver span of $19 \mathrm{~cm}$.

Oral prednisolone $\left(40 \mathrm{mg} \cdot \mathrm{day}^{-1}\right)$ was started and, within 2 weeks, the cough and diarrhoea were controlled, but on steroid tapering (a minimum daily dose of $20 \mathrm{mg}$ was needed for symptom control). Blood eosinophilia persisted at $\sim 1 \times 10^{9} \cdot \mathrm{L}^{-1}$.

Because of his persistent hypereosinophilia, he was referred for further haematological investigations. Bone marrow aspirate and trephine biopsy showed increased numbers of eosinophils and loosely clustered mast cells with no increase in blast cells or abnormal lymphoid infiltrate. There was no rearrangement of TCRB or TCRG genes to suggest lymphocyte clonality. Cytogenetic analysis was normal. Single-step RT-PCR for the FIP1L1-PDGFRA fusion gene was negative, but nested PCR was intermittently positive. Fluorescence in situ hybridisation (FISH) analysis showed CHIC2 deletion, suggesting the presence of this fusion gene, in $23 \%$ of interphase cells (normal: $\leqslant 10 \%$ ) (fig. 1). The weak positive results with RTPCR and the low proportion of abnormal cells on FISH analysis led to further molecular investigation, which revealed a breakpoint involving exon 16 of the FIP1L1 gene and exon 12

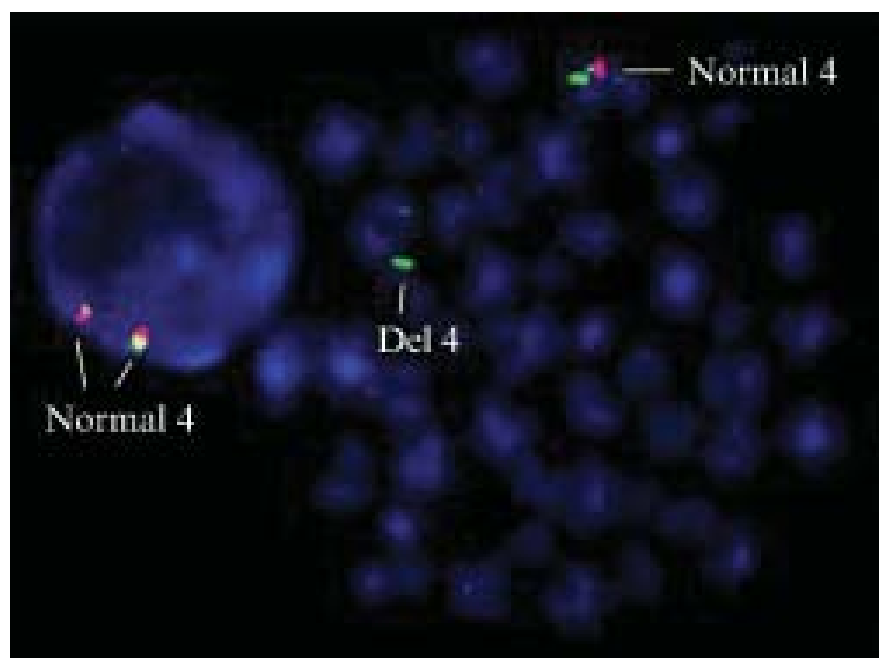

b)

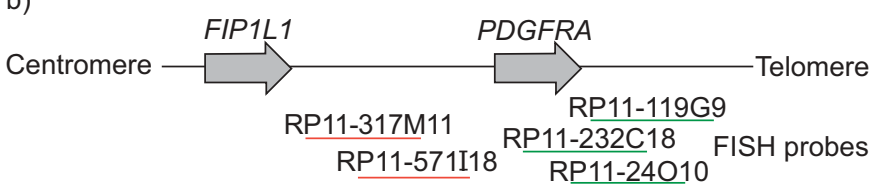

FIGURE 1. Fluorescence in situ hybridisation (FISH) analysis. a) Normal interphase with two overlapping red/green signals corresponding to two normal copies of chromosome 4 . The metaphase spread on the right shows one normal chromosome 4 and one CHIC2-deleted chromosome 4 (green signal only). b) Scheme indicating the position of the genes and FISH probes. of PDGFRA gene further downstream than reported previously (fig. 2). The position of this previously undescribed breakpoint probably explains the difficulty in detection of the fusion gene mRNA using standard PCR primers.

Following demonstration of the FIP1L1-PDGFRA fusion gene 10 months after starting prednisolone therapy, the tyrosine kinase inhibitor imatinib $\left(100 \mathrm{mg} \cdot \mathrm{day}^{-1}\right)$ was commenced, with a rapid disappearance of blood eosinophils. Prednisolone dosage was gradually discontinued after 2 months of imatinib therapy. The patient's cough has not recurred and his diarrhoea has improved since commencing imatinib. No side-effects have been noted and the fusion gene is no longer detectable by specific RT-PCR designed for the breakpoints present in this patient.

\section{DISCUSSION}

This patient had evidence of severe gastro-oesophageal reflux but his cough did not respond to treatment with proton pump inhibitors. A Nissen fundoplication that abolished reflux also failed to improve the patient's cough, contrary to what has been previously reported in patients in whom a cough was evidently the result of reflux [7]. This therefore excluded the possibility of gastro-oesophageal reflux as underlying his cough.

The association of the persistent hypereosinophilia with the cough was next investigated. There was evidence of eosinophil inflammation in the airways mucosa, but the patient did not have symptoms of asthma and his histamine bronchial responsiveness was not abnormal. The level of hypereosinophilia puts him into the category of idiopathic hypereosinophilic syndrome, but this diagnosis can now only be made after rigorously excluding all other causes of hypereosinophilia. Parasitic infestations, in view of his extensive travels to the tropics, were excluded. It was also unlikely that he represented a case of acute eosinophilic pneumonia, such as has been described in American servicemen serving in Iraq, attributed to exposure to desert sand or explosives [8].

Hypereosinophilic syndrome comprises a range of conditions, but recent developments have led to the identification of two distinct entities, in addition to residual idiopathic cases. First, a lymphocytic variant with hypereosinophilia secondary to a Tcell lymphoproliferative disorder demonstrated by the presence of a circulating clonal T-cells has been described $[9,10]$. This was excluded in this patient by the lack of rearrangement of the TCRB or TCRG genes. Secondly, a myeloproliferative disorder resulting from an interstitial deletion of the long arm of chromosome 4 , leading to formation of a FIP1L1-PDGFRA fusion gene and synthesis of a protein with aberrant tyrosine

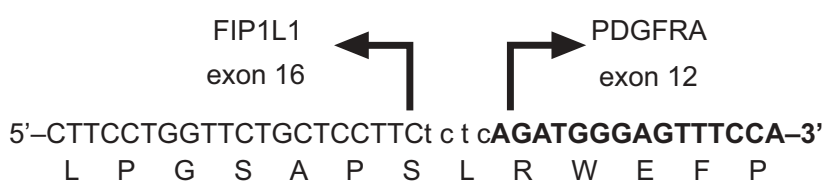

FIGURE 2. Molecular investigation of the FIP1L1-PDGFRA fusion in this case study, showing the in-frame mRNA junction and predicted amino acid sequence. The FIP1L1 sequence is shown in plain type, a four base pair insert of unknown origin in lower case and PDGFRA in bold type. 
kinase activity, has been described [6]. Since splenomegaly and gastrointestinal and pulmonary symptoms are recognised features of this syndrome, this patient was investigated in this regard and demonstrated the presence of the fusion gene. Other patients with the FIP1L1-PDGFRA syndrome have also shown bone marrow infiltration by cytologically abnormal mast cells [11]. Imatinib selectively inhibits ABL, PDGFR and KIT tyrosine kinases. FIP1L1-PDGFRA is particularly sensitive to imatinib and a low initial dose is therefore appropriate. The FIP1L1-PDGFRA syndrome may lead to death, either from end-organ (particularly cardiac) damage or from transformation to acute leukaemia. The striking response to imatinib therapy means that its correct identification and treatment is of critical importance to the patient. When the clinical suspicion is strong, atypical molecular results, as in this patient, are an indication for further investigation until the diagnosis is clarified.

This patient supports the concept that eosinophils may be the cause of cough. The role of eosinophils in the pathogenesis of asthma has been questioned following the recent observation that an anti-interleukin-5 monoclonal antibody had no effect on the late-phase response to allergen and bronchial hyperresponsiveness of asthma [12]. However, anti-interleukin-5 therapy did not deplete eosinophils completely in the airways mucosa and, also, other mediators may also be involved in eosinophilic activation. How eosinophils may induce cough requires further studies.

\section{REFERENCES}

1 Lalloo UG, Barnes PJ, Chung KF. Pathophysiology and clinical presentations of cough. J Allergy Clin Immunol 1996; 98: S91-S96.

2 Irwin RS, Madison JM. The diagnosis and treatment of cough. $N$ Engl J Med 2000; 343: 1715-1721.

3 Irwin RS, Curley FJ, French CL. Chronic cough: the spectrum and frequency of causes, key components of the diagnostic evaluation, and outcome of specific therapy. Am Rev Respir Dis 1990; 141: 640-647.

4 Niimi A, Nguyen LT, Usmani O, Mann B, Chung KF. Reduced $\mathrm{pH}$ and chloride levels in exhaled breath condensate of patients with chronic cough. Thorax 2004; 59: 608-612.

5 McGarvey LP, Heaney LG, Lawson JT, et al. Evaluation and outcome of patients with chronic non-productive cough using a comprehensive diagnostic protocol. Thorax 1998; 53: 738-743.

6 Cools J, DeAngelo DJ, Gotlib J, et al. A tyrosine kinase created by fusion of the PDGFRA and FIP1L1 genes as a therapeutic target of imatinib in idiopathic hypereosinophilic syndrome. N Engl J Med 2003; 348: 1201-1214.

7 Novitsky YW, Zawacki JK, Irwin RS, French CT, Hussey VM, Callery MP. Chronic cough due to gastroesophageal reflux disease: efficacy of antireflux surgery. Surg Endosc 2002; 16: 567-571.

8 Shorr AF, Scoville SL, Cersovsky SB, et al. Acute eosinophilic pneumonia among US Military personnel deployed in or near Iraq. JAMA 2004; 292: 2997-3005.

9 Simon HU, Plotz SG, Dummer R, Blaser K. Abnormal clones of $\mathrm{T}$ cells producing interleukin-5 in idiopathic eosinophilia. N Engl J Med 1999; 341: 1112-1120.

10 Brugnoni D, Airo P, Rossi G, et al. A case of hypereosinophilic syndrome is associated with the expansion of a CD3CD4+ T-cell population able to secrete large amounts of interleukin-5. Blood 1996; 87: 1416-1422.

11 Klion AD, Noel P, Akin C, et al. Elevated serum tryptase levels identify a subset of patients with a myeloproliferative variant of idiopathic hypereosinophilic syndrome associated with tissue fibrosis, poor prognosis, and imatinib responsiveness. Blood 2003; 101: 4660-4666.

12 Leckie MJ, ten Brinke A, Khan J, et al. Effects of an interleukin-5 blocking monoclonal antibody on eosinophils, airway hyper-responsiveness, and the late asthmatic response. Lancet 2000; 356: 2144-2148. 